\title{
Outflow from and asymmetries in the nebula around the LBV candidate Sk- $69^{\circ} 279$
}

\author{
K. Weis ${ }^{1,2, \star}$ and W. J. Duschl ${ }^{1,2, \star}$ \\ 1 Institut für Theoretische Astrophysik, Tiergartenstr. 15, 69121 Heidelberg, Germany \\ 2 Max-Planck-Institut für Radioastronomie, Auf dem Hügel 69, 53121 Bonn, Germany
}

Received 11 May 2000 / Accepted 27 June 2002

\begin{abstract}
We present and discuss new long-slit Echelle spectra of the LMC LBV candidate Sk $-69^{\circ} 279$ and put them in context with previous images and spectra. While at first glance it resembles a simple spherically expanding symmetric shell, we find a considerably more complex morphology and kinematics. The spectra indicate that morphologically identified deviations from sphericity are outflows of faster material out of the main body of $\mathrm{Sk}-69^{\circ} 279$. The morphological as well as the kinematic similarity with other LBV nebulae makes it likely that $\mathrm{Sk}-69^{\circ} 279$ is an LBV candidate and poses the question: in how far are outflows out of expanding LBV nebulae a general property of such nebulae - at least during some phases of their evolutions.
\end{abstract}

Key words. stars: evolution - stars: individual: Sk-69²79 - stars: mass-loss - ISM: bubbles - ISM: jets and outflows

\section{Introduction}

Stars are known up to masses around $100 M_{\odot}$ with main sequence luminosities of $10^{5-6} L_{\odot}$. As $O$ stars they are located in the upper left part of the Hertzsprung-Russell Diagram (HRD). In their later evolution, they leave the main sequence at almost constant luminosity towards redder spectral types, i.e., they quickly cool and become supergiants. However, instead of completing their evolution towards the red, the most massive ones among them enter a phase of very high mass loss (up to $10^{-4} M_{\odot} \mathrm{yr}^{-1}$ ) and reverse the direction of their evolution, i.e., the stars become hotter again (e.g., Schaller 1992; Langer et al. 1994; Schaerer 1996a,b). These stars are known as Luminous Blue Variables (LBVs). The location of the turning points of LBVs in the HRD is called the Humphreys-Davidson limit (HD-limit; Humphreys \& Davidson 1979, 1994; Langer et al. 1994). It is a function of the stellar luminosity. The position of most known LBVs and LBV candidates is illustrated in Fig. $1^{1}$, where a solid line marks the empirical HD-limit. Since LBVs also show spectral variability both the hottest and coolest temperature (e.g., their position in the non-eruptive and eruptive states) are indicated with filled and open dots. The regime

Send offprint requests to: $\mathrm{K}$. Weis, e-mail: kweis@mpifr-bonn.mpg.de

* Visiting Astronomer, Cerro Tololo Inter-American Observatory, National Optical Astronomy Observatories, operated by the Association of Universities for Research in Astronomy, Inc., under contract with the National Science Foundation.

1 This figure is nearly identical to Fig. 9 from Humphreys \& Davidson (1994), and was copied with the permission of the authors. of the hypergiants, red supergiants and the location of the mainsequence is shown for reference, as is the position of the precursor of SN1987A. Presently, only a few LBVs are known (roughly 40, including candidate objects, see, e.g., Humphreys \& Davidson 1994), of which 9 are in our Galaxy and 10 in the LMC.

The strong stellar winds and possible so-called giant eruptions lead to the formation of nebulae around LBVs, the $L B V$ nebulae (e.g., Nota et al. 1995; Weis 2001). These LBV nebulae are typically up to $2 \mathrm{pc}$ in diameter. Because of this small size, they can be studied only in our galaxy and - with the high resolution of the Hubble Space Telescope (HST) - in a few neighboring galaxies like, for instance, the Large Magellanic Cloud (LMC). For a better understanding of the evolution of LBVs and especially the formation of LBV nebulae a good knowledge of the parameters of the nebulae around LBVs is of great interest.

\section{The LBV candidate $S k-69^{\circ} 279$ and its nebula}

Sk-69 279 (Sanduleak 1970) is a bright and hot star $\left(m_{\mathrm{pg}}=\right.$ $12 \mathrm{~m} 5$ ) in the LMC in the field of the supergiant shell LMC 2, or - more precisely - on the eastern edge of the H II complex N 160 (see Henize 1956). The star was identified as a $\mathrm{H}_{\alpha}$ emission-line star, by Bohannan \& Epps (1974), and named $\mathrm{BE}(74) 619$ in this publication. The authors note in addition to the stellar emission-line a sharp feature seen bluewards of $\mathrm{H}_{\alpha}$, but do not discuss this feature further. Photometry of Sk-69 279 measured the blue supergiant with $m_{\mathrm{V}}=12$ m.79 


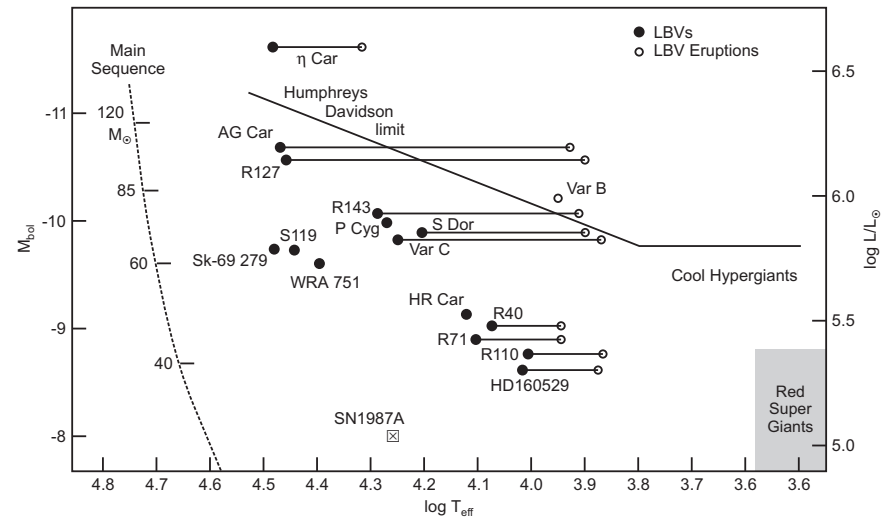

Fig. 1. HRD with the position of known LBVs and LBV candidates. The position of Sk-69 279 is indicated and similar to that of S119 or WRA 751, two LBV candidates. For reference the main sequence, the regime of the hypergiants and red supergiants and the position of the SN1987A precursor are plotted in addition. Sk-69 279 was positioned according to the temperature and luminosity determined by Thompson et al. (1982).

and $(B-V)=0$ m 05 (Isserstedt 1975). Later - based on prism spectroscopy - spectral types of O - B0 (Rousseau et al. 1978) and - based on grating observations - O9f (Conti et al. 1986) were quoted. Thompson et al. (1982) derived an effective temperature $T_{\text {eff }}=30300 \mathrm{~K}$, an absolute bolometric magnitude $M_{\text {bol }}=-9.72, M_{\mathrm{V}}=-6.77$ and a radius of $R=28 R_{\odot}$. Various analyses especially of UV data (IUE) and IR photometry of Sk-69 279 exist (Morgan \& Nandy 1982; Nandy et al. 1984, 1981; Gummersbach et al. 1995; Misselt et al. 1999) but were mainly used for the determination of the interstellar extinction towards the LMC and don't yield further information concerning the star itself, beside that it is reddened and that the stellar wind terminal velocity is of the order of $1000 \mathrm{~km} \mathrm{~s}^{-1}$ (Nandy et al. 1981, determined from the Si IV line). In a recent analysis of IUE spectra, Smith Neubig \& Bruhweiler (1999) found that $\mathrm{Sk}-69^{\circ} 279$ 's UV spectra resemble that of type B0 II, and note that the star shows weak Si IV, C IV, Al III, Fe III as well as strong Si II/III lines. The most recent photometry (Schmidt-Kaler et al. 1999) of Sk-69²79 finds values which are - within the errors - consistent with those of Isserstedt (1975).

Weis et al. (1995; in the following referred to as W95) found that $\mathrm{Sk}-69^{\circ} 279$ is surrounded by a circumstellar shell which has a diameter of about $18^{\prime \prime}$ or $4.5 \mathrm{pc}$ (assuming a distance to the LMC of $50 \mathrm{kpc}$ ). $\mathrm{H}_{\alpha}$ images of the shell (Weis et al. 1997, in the following referred to as W97) show a ring-like structure with some brighter areas within this ring (Knot E, Knot S and Knot N). Knot E reaches beyond the shell and clearly deforms the otherwise almost perfectly round ring structure. The shell is expanding very slowly with an expansion velocity $v_{\mathrm{exp}} \approx 14 \mathrm{~km} \mathrm{~s}^{-1}$ (W97). In addition to an otherwise almost spherical expansion (see, in particular, the flat expansion pattern in Fig. 4 in W97) kinematic deviations from a simple expanding shell were found. A feature, which was identified with the morphologically classified Knot E, moves $\sim 40 \mathrm{~km} \mathrm{~s}^{-1}$ faster than the center of expansion or about $20 \mathrm{~km} \mathrm{~s}^{-1}$ faster

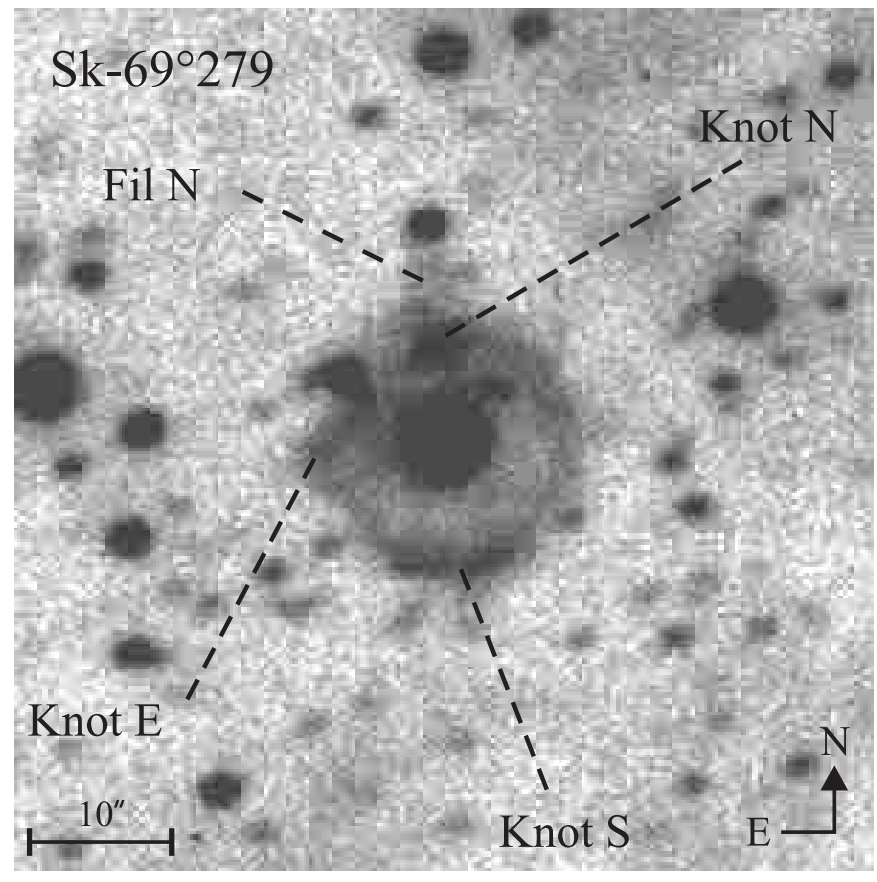

Fig. 2. Image of $\mathrm{Sk}-69^{\circ} 279$ and its nebula, taken with an $\mathrm{H}_{\alpha}$ filter and with sub-structure in the nebula marked (see also W97). The full image is $60^{\prime \prime} \times 60^{\prime \prime}$ large, north is up and east to the left.

than the backside of the shell at the corresponding position. One of the most unusual properties of the nebula around Sk $-69^{\circ} 279$ is the high $\left[\mathrm{N}_{\mathrm{II}}\right] \lambda 6583 \AA / \mathrm{H}_{\alpha}$ ratio, which reaches a value of 0.7 , compared to a $\left[\mathrm{N}_{\mathrm{II}}\right] \lambda 6583 \AA \mathrm{\AA} / \mathrm{H}_{\alpha}$ ratio of the background $\mathrm{H}_{\text {II }}$ region of only 0.07 . In particular due to the large $[\mathrm{N}$ II $] \lambda 6583 \AA / \mathrm{H}_{\alpha}$ ratio and the star's location in the HRD, W97 concluded that $\mathrm{Sk}-69^{\circ} 279$ actually is a good candidate for a LBV with a typical LBV nebula around it. LBV nebulae are characterized by such large $\left[\mathrm{N}_{\mathrm{II}}\right] \lambda 6583 \AA / \mathrm{H}_{\alpha}$ ratios due to CNO-processed material.

Supporting evidence for $\mathrm{Sk}-69^{\circ} 279$ being a candidate LBV came from the IUE analysis of Smith Neubig \& Bruhweiler (1999). They detected in Sk-69 279's spectra features which are found in and regarded as characteristic of the previously classified LBVs and LBV candidates. In a very detailed analysis of S Dor variables in the Galaxy and the Magellanic Clouds (van Genderen 2001), stars which show a certain varibility which is very typical for LBVs, Sk-69 279 appears among the ex-/dormant $S$ Dor variables ${ }^{2}$. In the same group as Sk-69 279 , van Genderen lists, for instance, S 119 , S 61 and He 3-519 - all stars known as LBV candidates (Humphreys \& Davidson 1994). Comparing the stellar parameters of Sk-69 279 with those of other LBVs, the star is only slightly hotter than most of the other LBVs as seen from Fig. 1. Sk-69 279 's location is, for example, still very similar to that of WRA 751 a galactic LBV candidate or to that of S 119 (again see Fig. 1), another LBV candidate and similar in temperature to it (see also van Genderen 2000, Table 8). Therefore the star's location in the HRD (see Fig. 1), its classification as

2 There is, however, no history of variability of Sk-69 279 so far, mainly due to the lack of data (van Genderen, priv. comm.). 
ex-/dormant S Dor variable and its [ $\mathrm{N}_{\text {II }}$ bright nebula makes it quite likely that $\mathrm{Sk}-69^{\circ} 279$ belongs to the LBV group. What we do know about the star is consistent with its LBV candidate classification. The star's slightly hotter temperature can probably be attributed to it being a more evolved LBV, as was proposed for He 3-519 (Davidson et al. 1993).

\section{Observation and data reduction}

\subsection{Imaging with the $0.9 \mathrm{~m}$ CTIO}

For the present investigations we made use of our already published image of Sk-69 279 and its nebula (W95, W97). The image was taken with the $0.9 \mathrm{~m}$ telescope at the Cerro Tololo Inter-American Observatory (CTIO). A $75 \AA$ wide $\mathrm{H}_{\alpha}$ filter, centered at $6563 \AA$ was used, which also contains emission from the [N $\left.\mathrm{N}_{\text {II }}\right]$ lines at $\lambda 6548$ and $6583 \AA$. The full field of view with this setup was $13.5 \times 13.5$ and the scale was about 0.4 pixel $^{-1}$. The exposure time was $600 \mathrm{~s}$, the seeing $\sim 1$.' 6 .

\subsection{Long-slit echelle spectroscopy}

To investigate the kinematics in the $\mathrm{Sk}-69^{\circ} 279$ nebula, new high-resolution long-slit echelle spectra were taken with the CTIO $4 \mathrm{~m}$ telescope. The setup of the echelle spectrograph was nearly identical to that used by W97: in both setups the cross-disperser was replaced by a flat mirror and a post-slit $\mathrm{H}_{\alpha}$ filter $(6563 / 75 \AA)$ was used to select the orders. The spectral region chosen contained the $\mathrm{H}_{\alpha}$ line and the two [NII] lines at $\lambda 6548$ and $6583 \AA$. The spatial slit length was vignetted to $\sim 4^{\prime}$ and we used the $791 \mathrm{~mm}^{-1}$ echelle grating with a slitwidth of $150 \mu \mathrm{m}$ (in contrast to W97, who used a slit width of $250 \mu \mathrm{m}$ ). This leads to an instrumental FWHM at the $\mathrm{H}_{\alpha}$ line of $\sim 8 \mathrm{~km} \mathrm{~s}^{-1}$. The data were recorded with the long focus red camera and a $2048 \times 2048$ pixel squared $C C D$, with a pixel size of $0.08 \AA \mathrm{pixel}^{-1}$ along the dispersion and $0.26 \mathrm{pixel}^{-1}$ on the spatial axis. The spectra were taken on January 29, 1999. The seeing was $\sim 1$ '. 2 during the observations. The weather was not photometric. For wavelength calibration and geometric distortion correction, thorium-argon comparison lamp frames were taken. The telluric lines visible in the spectra helped to improve the absolute wavelength calibration. The final accuracy of the wavelength calibration is estimated to be at least $0.04 \AA$ or about $2 \mathrm{~km} \mathrm{~s}^{-1}$.

The data reduction was perfomed using the longslit package in $\mathrm{IRAF}^{3}$. For wavelength calibration a list of thorium and argon lines (3180-9665 $\AA$, as pre-installed in IRAF and revised in Feb. 1992, Daryl Willmarth, see IRAF help on linelists) was used, and a Chebychev 6th order fit. For the correction of the deformation along the spatial axis, frames with stars present were used. In this case, six stars were available, located at different positions on the CCD. Here a 2nd order Chebychev function was fitted. After fitting the calibration files, this database was used and transformed onto the data. After correcting for the

3 IRAF is distributed by the National Optical Astronomy Observatories which is operated by AURA, Inc. under cooperative agreement with the NSF.

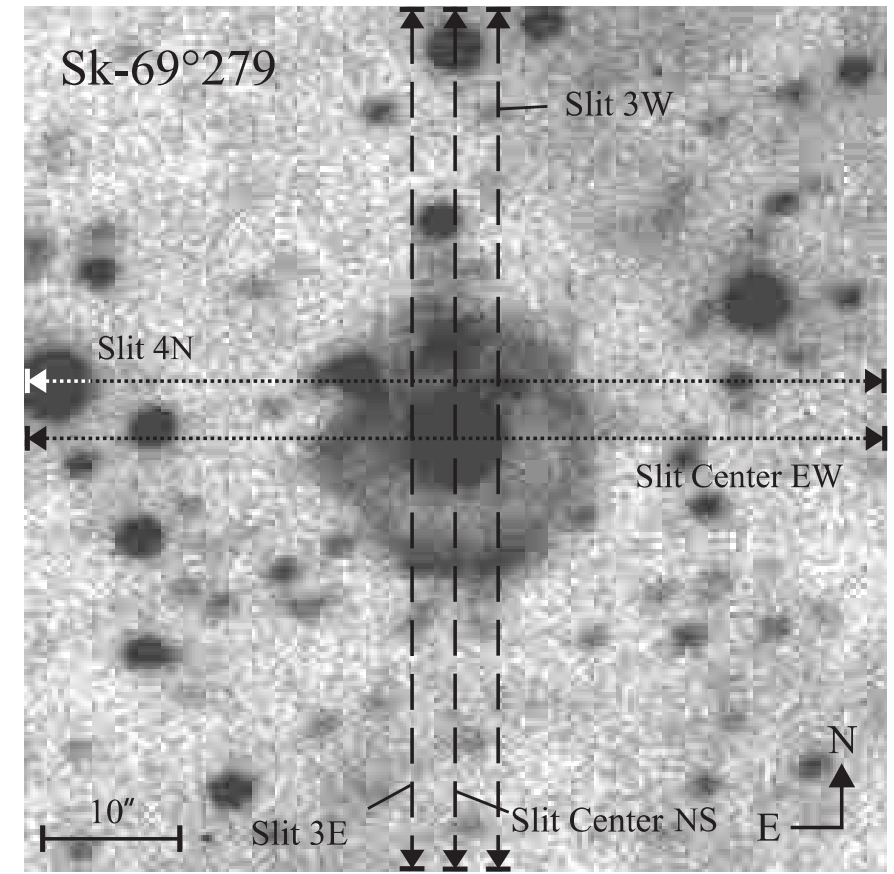

Fig. 3. Here the same images as in Fig. 2 is shown of Sk-69 279 now with the orientation of the slits and marked with the corresponding names.

distortion along the spatial axis and the wavelength calibration, the final fitting of the data, e.g., the determination of the velocities, was performed using a Gauss function, and correcting for the continuum emission. The accuracy to which the peak of the Gaussian could be fitted to the data (that is the central velocity) was estimated fitting all lines present and comparing the offsets (line fits to each individual line were always identical within the limits of the fitting accuracy which is smaller than $0.2 \mathrm{~km} \mathrm{~s}^{-1}$ ). The errors of the radial velocity measurements are therefore estimated to be of the order of $\pm 0.5 \mathrm{~km} \mathrm{~s}^{-1}$. Since this is much smaller than the size of the symbols used in all position-velocity diagrams ( $p v$-diagrams), no error bars are visible in these plots.

To improve on the spatial coverage of our spectral data, we chose a position angle (PA) of $180^{\circ}$, i.e., orthogonal to that of the spectra by W97. At this PA, three spectra were taken: one centered on the star (Slit Center NS) and two offset $3^{\prime \prime}$ to the east (Slit $3 E$ ) and to the west (Slit $3 W$ ) of the central star, respectively. The new slit positions as well as the ones from W97 (dotted lines) are indicated and labeled in Fig. 3. For consistency we label W97's spectra as Slit $4 N$ and Slit Center EW. They were published originally as Figs. $3 \mathrm{a}$ and $3 \mathrm{~b}$ in W97. The echellograms of all five spectra are shown in Fig. 4. The echellograms are $1^{\prime}$ in spatial direction and centered on the star, or - in cases of the off-centered slits - on the projected position of the star onto the slit. The spectral range of each echellogram is $65 \AA$ and the central position coincides with the rest wavelength of $\mathrm{H}_{\alpha}$. In Fig. 5 we present the corresponding $p v$ diagrams of the respective slits. Velocities are quoted in the heliocentric reference system with the systemic velocity subtracted, and are determined from the Doppler shifts in the [N II] $\lambda 6583 \AA$ line. 


\section{The morphology of the nebula around Sk-69 279}

A morphological discussion has still to be based on the image discussed in W97 as no improvement was achieved since. W97 noticed three deviations in Sk-69²79's nebula from a spherical shell, manifesting themself as extended knots (see also Fig. 2) and named Knots E, S, and N. Knots N and S are located within the ring structure and are characterized by their higher surface brightness as compared to their surroundings. In contrast, Knot E manifests itself as a geometrical deviation from the ring structure appearing as a triangularly shaped attachment to the ring. To disentangle these knots from possible back/foreground stars a continuum subtracted image was made and shown in W97. While the emission (i.e., the continuum) of all stars in the field was successfully subtracted, all identified knots still show up in the continuum free image, giving evidence that the knots are real brighter emission line regions within the shell. In addition to these already described features, we identify in Sk-69 279 's nebula the Filament N (Fil N, Fig. 2). Fil N extends from Knot N about 7'. 1 (corresponding to $1.7 \mathrm{pc}$ ) to the north and is - similar to Knot E roughly triangular in shape. Fil $\mathrm{N}$ has a very low surface brightness compared to other parts of the nebula and might easily be mistaken as a part of the background $\mathrm{H}$ II region. Because of this, its membership to Sk-69 $279^{\prime}$ 's nebula can be established only with the help of a spectral analysis (Sect. 5) and thus could not be classified earlier.

\section{The kinematics of the nebula around Sk- $69^{\circ} 279$}

W97's two spectra of the nebula around Sk-69 279 have already shown that the expansion is not perfectly spherical but displays faster moving small scale features. The three new spectra taken with a north-south orientation support these earlier results. Figure 4 shows the echellograms of all five spectra. Expansion ellipses are clearly visible main features in all spectra.

For comparison purposes, we model the expansion as being spherically symmetric with a velocity $v_{\exp }$ and a radius $R$ of the nebula. We introduce a coordinate system $\{\eta, \xi\}$, where the coordinate $\xi$ is measured along the slit and has its origin at the star's projected position, and the coordinate $\eta$ in a perpendicular direction with the same origin. The observed radial velocity distribution $v_{\text {rad,rel }}^{\text {spher }}$ relative to the systemic velocity then is

$\left|v_{\text {rad,rel }}^{\text {spher }}(\eta, \xi)\right|=v_{\exp }\left(\frac{R^{2}-\eta^{2}-\xi^{2}}{R^{2}}\right)^{1 / 2}$.

In Fig. 5 we compare for all five slit positions and orientations the model velocities with the observed values. The measured radial velocities were transformed into the central star's reference system by correcting for a systemic velocity of $230 \mathrm{~km} \mathrm{~s}^{-1}$ (W97). We find that in all cases the observed run of the radial velocities differs from what a pure spherical model predicts. These differences are significant given the accuracy of our spectra.

In addition, a closer comparison of the pv-diagrams (Fig. 4) shows that the maximum expansion velocities of each Doppler ellipse lie at different positions in each slit. In Slit 3E the maximum expansion velocity amounts to $15.1 \mathrm{~km} \mathrm{~s}^{-1}$ at the position $0^{\prime \prime}$, in Slit Center NS it is $14.8 \mathrm{~km} \mathrm{~s}^{-1}$ at $5^{\prime \prime} .7$, in Slit $3 \mathrm{~W}$ $17.5 \mathrm{~km} \mathrm{~s}^{-1}$ at 3 .' 7 , in Slit Center EW $16.0 \mathrm{~km} \mathrm{~s}^{-1}$ at $-4^{\prime \prime}$, and in Slit $4 \mathrm{~N} 16.9 \mathrm{~km} \mathrm{~s}^{-1}$ at $2^{\prime \prime}$. These off-centered maximum expansion velocities and the asymmetric shapes of the ellipses are a clear indication that the expansion is not spherically symmetric. In particular in Slit 3W (see middle panel in Figs. 4 and 5 ) the expansion pattern is deformed and considerably deviates from an elliptic shape, being smaller at the center (see waistlike deformation at position $0^{\prime \prime}$ ) and getting wider to the north at $\sim 4^{\prime \prime}$.

Comparing the positions of the maximum expansion velocity of each slit we find that the regions with the highest expansion velocities are located nearly exactly to the north (since the maximum expansion velocity found in the north-south oriented slits lie at positive offsets) and to the east of the star. Two of the spectra (Slit 3E and Slit 4N) have expansion ellipses that are open on one side, in Slit $3 \mathrm{E}$ to the north (upper part) and in Slit $4 \mathrm{~N}$ to the east (lower part). Here the shell forms no coherent structure anymore, or only very faint emission with a much lower surface brightness (below our detection limit) is present.

Beside of this global expansion pattern, several regions are found in the spectra which move significantly faster or slower than the expansion ellipse at that point or extend beyond the ellipse. Most prominent is a feature seen in Slit Center NS (Figs. 4 and 5). Here to the north (top) of the slit a long red-shifted emission extends beginning at the end of the ellipse. This feature is about $10^{\prime \prime}$ long, corresponding to $2.5 \mathrm{pc}$. The spectrum shows that with increasing distance the velocity becomes larger and increases from the edge of the shell at $6.0 \mathrm{~km} \mathrm{~s}^{-1}$ by about $15 \mathrm{~km} \mathrm{~s}^{-1}$ to $21.0 \mathrm{~km} \mathrm{~s}^{-1}$ at our outermost measurement. This faster moving structure can be identified with the morphologically classifies Filament N. A corresponding fainter emission can be traced in Slits $3 \mathrm{E}$ and $3 \mathrm{~W}$ to the north end of the expansion ellipses. This emission is also a contribution of Filament N, which is partly intercepted by these slits (see position Slit 3W, Slit 3E in Fig. 3). Also at the northern rim (at $\sim 6^{\prime \prime}$ ) of the ellipse in Slit Center NS appears a structure which shows a brighter knot-like emission and is blue-shifted with respect to the expansion ellipse at that position. The highest blue-shifted emission of the structure is at about $-31.0 \mathrm{~km} \mathrm{~s}^{-1}$ and thus $20 \mathrm{~km} \mathrm{~s}^{-1}$ faster than the expanding shell. The higher surface brightness and the position makes it likely that this blue-shifted emission results from Knot $\mathrm{N}$. At the south end ( -10 to $\left.-15^{\prime \prime}\right)$ of Slit Center NS and Slit $3 \mathrm{~W}$ we detect a component moving with approximately $+10 \mathrm{~km} \mathrm{~s}^{-1}$ (Fig. 4) which is therefore expanding faster than the shell at this point. This component can be assigned to the optically identified structure called Knot S. Finally we note that Slit Center EW has a highly red-shifted knot at $+42 \mathrm{~km} \mathrm{~s}^{-1}$ and was already identified with Knot $\mathrm{E}$ in W97.

\section{Discussions and conclusions}

The images of the nebula around Sk $-69^{\circ} 279$ as well as the spectra show several cases of deviations from the morphology 
[N II] 6548

$\mathrm{H}$

[N II] 6583
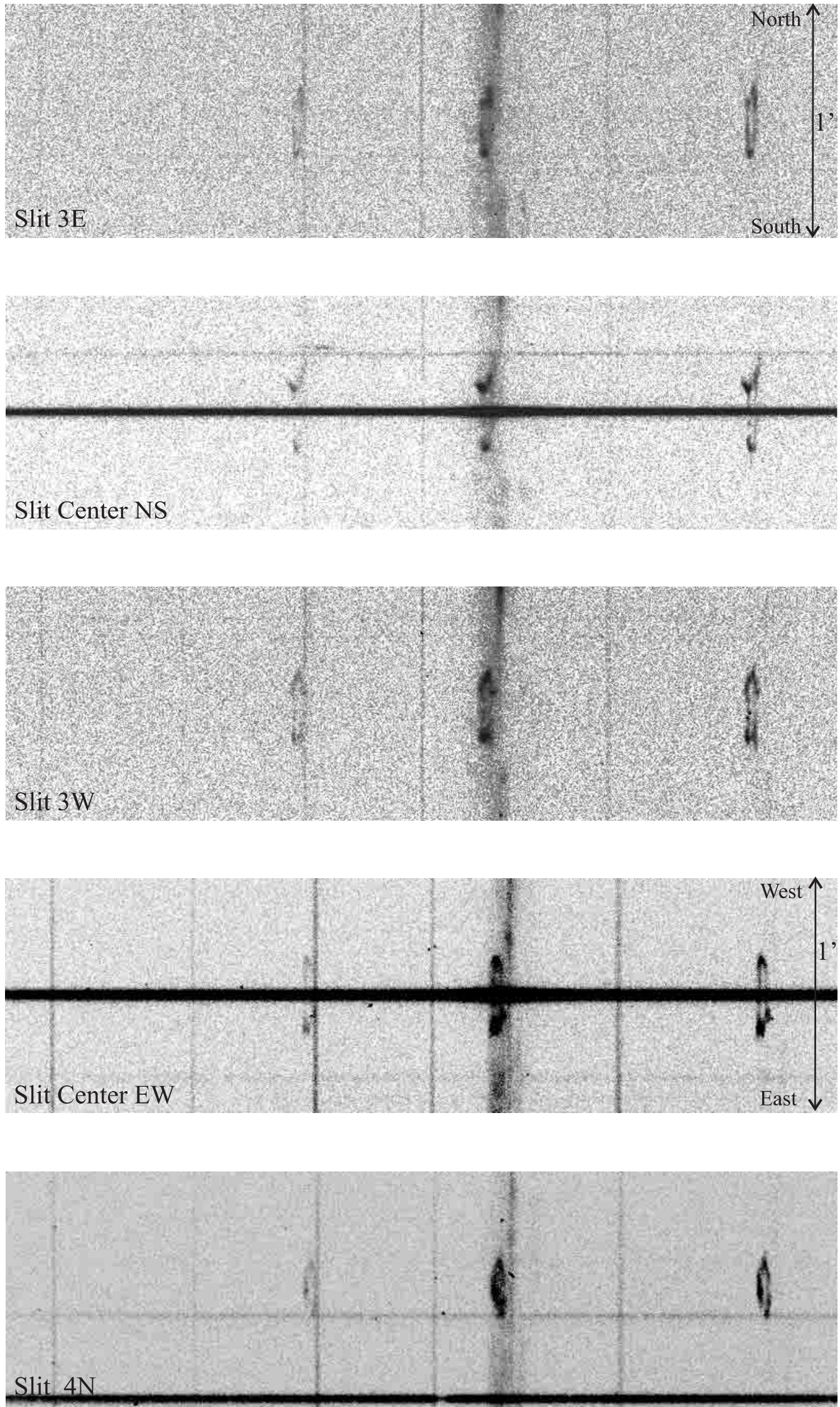

Fig. 4. Echelle spectra for all five slit positions and orientations. The slit length is $1^{\prime}$, the slit positions and orientations are shown in Fig. 3. 

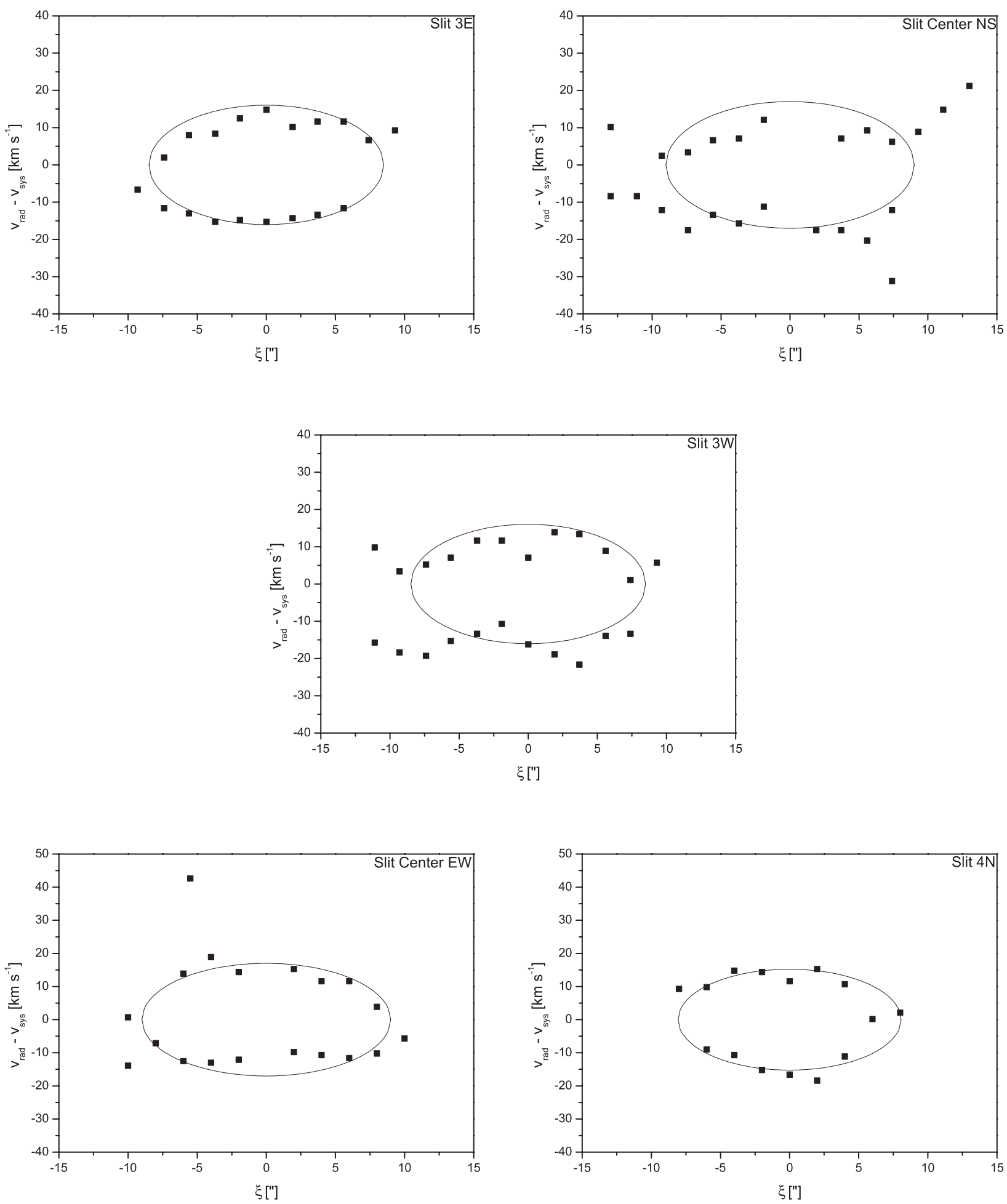

Fig. 5. Position-velocity diagrams for all five spectra of Fig. 4. The lines give the model results according to Eq. (1), while the squares give the corresponding measurements for a systemic velocity of $230 \mathrm{~km} \mathrm{~s}^{-1}$. The coordinate $\xi$ is measured along the slit with the projected position of the star at the origin. Negative offsets are to the south (Slits Center NS, 3W and 3E) or to the east (Slits Center EW, 4N).

and kinematics of a spherical nebula with symmetric expansion.

Due to the lack of sufficiently resolved images of the nebula, one can only speculate - with the help of spectra - about the three dimensional structure of the nebula. While part of its main body may reasonably well be approximated by a sphere with a symmetric expansion, the nebula is expanding faster in the north-eastern hemisphere comparing, for instance the higher expansion velocities detected in Slits $3 \mathrm{~W}\left(17.5 \mathrm{~km} \mathrm{~s}^{-1}\right)$ and Slit $4 \mathrm{~N}\left(16.9 \mathrm{~km} \mathrm{~s}^{-1}\right)$ with those in the nebula's center 
(typically $15 \mathrm{~km} \mathrm{~s}^{-1}$, Slit $3 \mathrm{E}$ or Slit Center NS) or the southern part of the nebula which expands even slower than the center (see $p v$-diagrams in Fig. 5). Even though the difference of the expansion velocities is marginal, it is significantly higher than the expected errors, and therefore most likely real.

Knot E shows an extension of the nebula of about 3"'2 $(0.8 \mathrm{pc})$ and moves faster than the shell (red-shifted). Here material seems to surpass the shell and move away from the observer. Similarly, the material of Knot N approaches us faster than most of the shell (see blue-shifted extension in Slit Center NS). In addition to this faster moving material of Knot N, there gas is streaming out. This out-streaming gas as traced in the spectra - corresponds to the morphologically identified Fil N. Fil $\mathrm{N}$ has a length of roughly half the diameter of the shell and moves up to $15 \mathrm{~km} \mathrm{~s}^{-1}$ faster along the lineof-sight. Throughout the nebula - including Fil $\mathrm{N}-$ we find a $\left[\mathrm{N}\right.$ II] $\lambda 6583 \AA / \mathrm{H}_{\alpha}$ ratio in the range of $0.65-0.7$ and thus further evidence for Fil $\mathrm{N}$ being a part of Sk-69 279 's nebula.

The spectra also indicate that to the north-east the shell of the nebula around $\mathrm{Sk}-69^{\circ} 279$ is not closed. Together with the faster moving Fil $\mathrm{N}$ it is therefore most likely that the nebula around $\mathrm{Sk}-69^{\circ} 279$ shows an outflow. The north-eastern part of the nebula has opened up and material is streaming out evidenced in particular by Fil N. Both properties, open Doppler ellipses and an increasing velocity extension, were also found in the nebula around the LMC LBV candidate S 119 (Weis et al. 2002). The kinematics and morphology of the nebula around S 119 are very similar to those found here. In particular, it is worthwhile noting that this outflow from Sk-69'279's nebula, shows the same linear increase in velocity as the outflow in S 119. The out-streaming material moves the faster the further it is away from the star. Comparing both objects we conclude that the nebulae of these stars are quite similar and represent (candidate) LBV nebulae with outflows.

It is not clear whether Knot $\mathrm{E}$ is also part of an outflow or if this feature resembles more an asymmetry in the nebula comparable to the Caps in WRA 751 (Weis 2000) or R 127 (Weis 2002 , in prep.). An interpretation as an outflow or as caps is supported by the faster moving extension in Slit $3 \mathrm{~W}$.

The dynamic age of a nebula is defined by $\tau=\eta\left(r / v_{\exp }\right)$, with $\eta$ depending on whether we model a steady fast wind swept up bubble ( $\eta=1$, García-Segura \& Mac Low 1995), an energy-conserving bubble $(\eta=0.6$, Weaver et al. 1977) or a momentum-conserving bubble $(\eta=0.5$, Steigman et al. 1975). For $\mathrm{Sk}-69^{\circ} 279$ this would yield a range for the dynamic age between $6.3 \times 10^{4}$ (for a maximum expansion velocity of $17.5 \mathrm{~km} \mathrm{~s}^{-1}$ and a momentum conserving bubble) to $1.5 \times 10^{5}$ years (with the slowest expansion of $14 \mathrm{~km} \mathrm{~s}^{-1}$ and steady sweep-up). Compared to dynamic ages of other LBV nebula (ranging typically between $0.01-210^{4}$, see, e.g., Nota et al. 1995) this value is up to a factor of 10 higher than the average. With the estimated duration of the LBV phase of about 25000 years (Maeder \& Meynet 1987; Humphreys \& Davidson 1994; Bohannan 1997) the dynamic age would be too high for the nebula being created during the star's LBV phase. In this context, however, one has to keep in mind that the duration of the LBV phase is mainly estimated by determining the ratio of LBVs stars to Wolf-Rayet stars, i.e., a method with comparatively large inherent errors, due to incompleteness of the respective samples. In the context of Sk $-69^{\circ} 279$ beeing a nebula with an outflow, as proposed here, these age determinations with the help of the nebula's size and expansion velocity might well be overestimated. If the nebula was disrupted by the outflow, most likely the expansion velocity of the nebula decreased since the pressure dropped. Therefore the dynamic age determination yields a higher age, as a result of the lower expansion velocity. The dynamic age for S 119, another LBV candidate showing outflow, for instance, also seems to be slightly higher compared to other LBV nebulae, reaching a maximum of $\sim 4 \times 10^{4}$ years (Weis et al. 2002).

While from the kinematics alone it is not yet clear in each case which features are part of an outflow and which are caplike extensions of the shell, the structure of the expansion ellipses as well as Fil $\mathrm{N}$ show that at least these features are due to an outflow. The similarity with other LBVs and their nebulae makes it likely that $\mathrm{Sk}-69^{\circ} 279$ is at least a good candidate LBV and poses the question how far outflows out of expanding LBV nebulae are a general property of such nebulae - at least during some phases of their evolution - and thus connects directly to the question of their origin and evolution.

Acknowledgements. We thank the referee for helpful comments on the manuscript. KW thanks Dr. D. J. Bomans (Bochum) for many helpful discussions on the subject of this paper. Sincere thanks go to Prof. R. M. Humphreys for permitting us to reprint and slighlty vary Fig. 9 from Humphreys \& Davidson (1994). The data reduction and analysis was in part carried out on a workstation provided by the Alfried Krupp von Bohlen und Halbach-Stiftung to the ITA, Heidelberg. Their support is gratefully acknowledged.

\section{References}

Bohannan, B. 1997, in Luminous Blue Variables: Massive Stars in Transition, ed. A. Nota, \& H. J. G. L. M. Lamers, ASP Conf. Ser., 120,3

Bohannan, B., \& Epps, H. W. 1974, A\&AS, 18, 47

Conti, P. S., Garmany, C. D., \& Massey, P. 1986, AJ, 92, 48

Davidson, K., Humphreys, R. M., Hajian, A., \& Terzian, Y. 1993, ApJ, 411,336

García-Segura, G., \& Mac Low, M.-M. 1995, ApJ, 455, 145

Gummersbach, C. A., Zichgraf, F.-J., \& Wolf, B. 1995, A\&A, 302, 409

Henize, K. G. 1956, ApJS, 2, 315

Humphreys, R. M., \& Davidson, K. 1979, ApJ, 232, 409

Humphreys, R. M., \& Davidson, K. 1994, PASP, 106, 1025

Isserstedt, J. 1975, A\&AS, 19, 259

Langer, N., Hamann, W.-R., Lennon, M., et al. 1994, A\&A, 290, 819

Maeder, A., \& Meynet, G. 1987, A\&A, 182, 243

Misselt, K. A., Clayton, G. C., \& Gordon, K. D. 1999, ApJ, 515, 128

Morgan, D. H., \& Nandy, K. 1982, MNRAS, 199, 979

Nandy, K., Morgan, D. H., Willis, A. J., Wilson, R., \& Gondhalekar, P. M. 1981, MNRAS, 196, 955

Nandy, K., Thompson, G. I., Morgan, D. H., \& Houziaux, L. 1984, MNRAS, 210, 131

Nota, A., Livio, M., Clampin, M., \& Schulte-Ladbeck, R. 1995, ApJ, 448, 788

Rousseau, J., Martin, N., Prevot, L., et al. 1978, A\&AS, 31, 243

Sanduleak, N. 1970, Contr. Cerro-Tololo Obs., 89, 1

Schaerer, D., de Koter, A., Schmutz, W., \& Maeder, A. 1996a, A\&A, 310, 837 
Schaerer, D., de Koter, A., Schmutz, W., \& Maeder, A. 1996b, A\&A, 312,475

Schaller, G., Schaerer, D., Meynet, G., \& Maeder, A. 1992, A\&AS, 96,269

Schmidt-Kaler, T., Gochermann, J., Oestreicher, M. O., et al. 1999, MNRAS, 306, 279

Smith Neubig, M. M., \& Bruhweiler, F. C. 1999, AJ, 117, 2856

Steigman, G., Strittmatter, P. A., \& Williams, R. E. 1975, ApJ, 198, 575

Thompson, G. I., Nandy, K., Morgan, D. H., et al. 1982, MNRAS, Weis, K., Duschl, W. ApJ, 218, 377 325,1157 (W97) van Genderen, A. M., A\&A, 366, 508

Weaver, R., McCray, R. A., Castor, J., Shapiro, P., \& Moore, R. 1977,

Weis, K. 2000, A\&A, 357, 938

Weis, K. 2001, Rev. Mod. Astron., 14, 261

Weis, K., Bomans, D. J., Chu, Y.-H., Joner, M. D., \& Smith, R. C. 1995, Rev. Mex. Astron. SdC, 3, 237 (W95)

Weis, K., Chu, Y.-H., Duschl, W. J., \& Bomans, D. J. 1997, A\&A, 200,551 